\title{
An Appreciative Approach to Burnout
}

\author{
Richard B. Gunderman, MD, PhD \\ Department of Radiology, Indiana University \\ 702 North Barnhill Drive, Room 1053 \\ Indianapolis, IN 46202
}

We need a new way of approaching burnout. Like all medical specialists, radiologists report that we are burning out, and a recent survey reveals that radiology now ranks 7th among all specialties (up from 20 th), with $45 \%$ of radiologists reporting that they are burned out (1). Broadly speaking, this means that radiologists are experiencing worsening emotional exhaustion, depersonalization, and inefficacy (2). The fact that the burnout situation in radiology is deteriorating suggests that we need to look for new ways to address this challenge.

Addressing burnout represents an especially important mission for those involved in educating the next generation of radiologists. Medical students, residents, fellows, and newly qualified radiologists need to master core knowledge and skills, but they also need to develop habits that enable them to find fulfillment in their careers and build and sustain the kinds of organizations that promote it. By teaching more effective approaches to burnout, we can place the profession's future on a firmer foundation.

\section{Appreciative Inquiry}

One such approach is appreciative inquiry, a model of organizational development introduced in the late 1980s (3). As opposed to problem-based models, which sometimes exacerbate the very difficulties they seek to address, appreciative inquiry attempts to shift the conversation to the best in what is happening, with the aim of accentuating and building on it. If the proponents of appreciative inquiry are correct,

This is the author's manuscript of the article published in final edited form as:

Gunderman, R. B. (2019). An Appreciative Approach to Burnout. Academic Radiology, 26(8), 1118-1119. https://doi.org/10.1016/j.acra.2019.03.005 
radiologists need to stop focusing exclusively on "What is broken?" and "What needs to be fixed?" and instead ask, "What brings us pride and a sense of fulfillment?"

Appreciative inquiry assumes that what we do is founded on what we believe to be true, both individually and together. If we can enhance our daily conversations - both intrapersonal and interpersonal - we can improve our work. We are continually reshaping our work and organizations by the questions we ask about them, and asking better ones can take us in better directions. In a sense, every job and every organization has a story, and by changing these stories, we can both do better work and derive more fulfillment from it.

In other words, if we talk mainly about the bleak future that awaits us, over time we will become increasingly consigned to it, dulling our imaginations, draining our enthusiasm, and depriving ourselves of hope. On the other hand, if we can focus on the causes for hope and their enhancement, the outlook will brighten. By focusing on what we do or could do best, we can augment openness to change, energy levels, and creativity. We can turn a dark and depressing narrative into one that offers real hope, revitalizing both our work and the organizations in which we do it.

\section{The Discourse of Deficit}

Before turning to appreciative inquiry's solution, it is important to understand in more depth how focusing on burnout often merely exacerbates the problem (4). Not surprisingly, many discussions of burnout tend to be focused on deficits and problems. They are dominated by sentiments such as "We are not measuring up," "We are performing poorly," and "Things around here are getting more and more discouraging." We begin to think of ourselves as suffering from something approaching a mental illness, which undermines our sense of fulfillment.

A substantial difficulty with much discourse around burnout is the supposition that we lack the resources necessary to solve the problem. Too often, we suppose that radiologists and radiology organizations need help from outside experts. The implicit assumption is this: so long as radiologists and radiology 
organizations have only themselves to rely on, we are doomed. Unfortunately, this only exacerbates the sense of inefficacy that lies at the core of burnout.

This is one example of how challenges can be elevated to the level of evidence of a deep and pervasive pathology. The fact that work is sometimes frustrating confirms emotional exhaustion. The fact that radiologists sometimes feel isolated is taken as evidence that our work is depersonalized. And the fact that we cannot instantly remove such obstacles is taken as proof that we are powerless. In other words, a relentlessly deficit-focused discourse tends to deepen the very deficits it describes.

As such findings get disseminated both within and outside the organization, a profession-wide narrative of crisis begins to develop. More and more presentations, articles, and casual conversations focus on how desperate things are, functioning as self-fulfilling prophecies and accelerating the pace of descent.

Over time, the focus on burnout exerts a greater and greater training effect. We learn how to talk about burnout, becoming increasingly familiar with its symptoms and signs, its adverse effects, and the difficulties associated with remedying it. And in learning more and more about burnout, we get better and better at burning out. In some cases, cultures can become infected with the cynical notion that it is socially unacceptable or simply "uncool" to be caught taking pride or finding joy in work.

None of this is to say that burnout is nonexistent, a mere figment of our imaginations. Many radiologists have legitimate reasons to be discouraged. The point is not that our problems exist only in our minds, but rather that an exclusively problem-focused approach often exacerbates them. The point is not that we should ignore what is not working and pretend "All is for the best in the best of all possible worlds," but that we need to give more attention to what is good in our work.

\section{An Appreciative Response}

Appreciative inquiry urges us to stop seeing burnout as a kind of mental illness begging for a cure, and instead to focus on the sources of joy and fulfillment in work.(5) By shifting our conversations to what works well, we can find new opportunities to improve. What we know and believe are not merely the 
products of empirical data, but rather created through conversation. What we think we know is often as important as anything we can justify in evidence-based terms. By shifting to strengths, we can build hope.

Burnout tends to be a narrative of isolation, but when we come together to talk about what makes work and life meaningful, we promote community. Appreciative inquiry is inherently collaborative - its methodology rests on having some members of an organization talk with other members about the things that make them proud, fill them with joy, and give them a sense that they are really coming to life. The simple act of discussing such experiences builds a shared sense of what is best about work and what we can accentuate going forward.

The fact that appreciative inquiry focuses on what is working well might suggest that it is inherently conservative, tending to promote and preserve the status quo. This is not necessarily so. In fact, by helping us to see what matters most to us about work, it helps to illuminate a path of change toward more meaning and fulfillment. For example, radiologists who discover that face-to-face consultation provides the most fulfillment might try to reorganize work to promote more such interactions.

The point is this: we can best address the conditions that underlie burnout - and thereby avoid burning ourselves up - not primarily by delving deeper and deeper into problems, but by treating our work and the organizations in which we do it as sources of learning and inspiration. We often have everything we need to do better; we just need to get started on the kinds of conversations that will help us recognize and draw fully on it. 


\section{References}

1. Kaplan DA. Stop burnout in radiology before it starts. Diagn Imaging. 2018; 27 February

Available at: http://www.diagnosticimaging.com/di-executive/stop-burnout-radiology-it-starts.

2. Maslach C, Leiter MP. Understanding the burnout experience: recent research and its implications for psychiatry. World Psychiatry 2016; 15:103-111.

3. Cooperrider DL, Whitney D. A positive revolution in change. In: Cooperrider DL, Sorenson PF, Whitney D, Yaeger TF, eds. Appreciative inquiry: an emerging direction for organization development, Champaign, IL: Stipes; 2001:9-29.

4. Gergen KJ. Toward transformation in social knowledge. New York: Springer 1982.

5. Ludema JD. From deficit discourse to vocabularies of hope: the power of appreciation. In:

Cooperrider DL, Sorenson PF, Whitney D, Yaeger TF, eds. Appreciative inquiry: rethinking human organization toward a positive theory of change, Champaign, IL: Stipes 2000:265-287. 